Document downloaded from:

http://hdl.handle.net/10251/68383

This paper must be cited as:

Palanca Cámara, J.; Heras Barberá, SM.; Botti Navarro, VJ.; Julian Inglada, VJ. (2014). receteame.com: a Persuasive Social Recommendation System. En Advances in Practical Applications of Heterogeneous Multi-Agent Systems. The PAAMS Collection. Springer. 367370. doi:10.1007/978-3-319-07551-8_40.

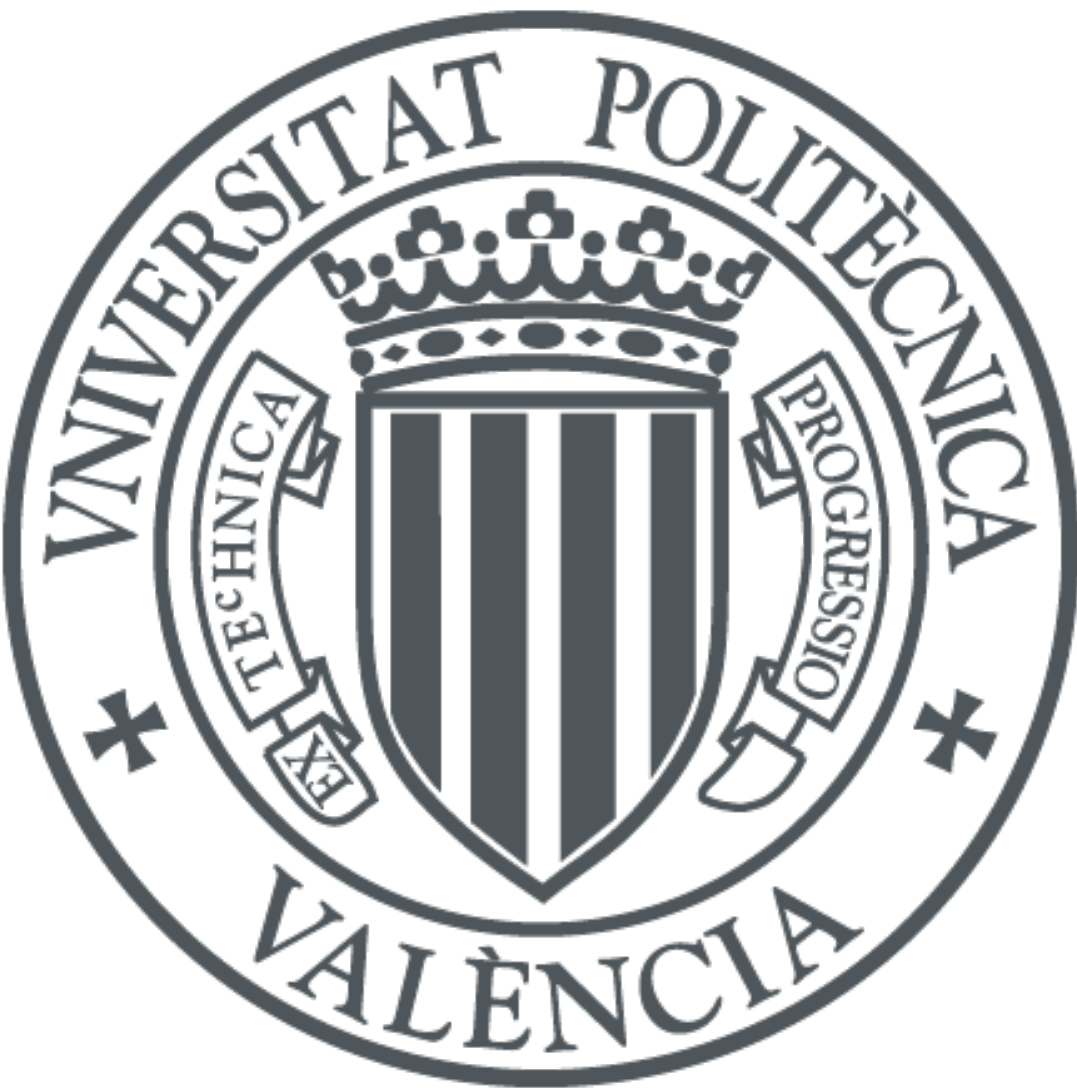

The final publication is available at

http://link.springer.com/chapter/10.1007\%2F978-3-319-07551-8_40

Copyright Springer

Additional Information

The final publication is available at Springer via http://dx.doi.org/10.1007/978-3-319-07551840 


\title{
receteame.com: a Persuasive Social Recommendation System
}

\author{
Javier Palanca, Stella Heras, Vicente Botti, Vicente Julián \\ Departamento de Sistemas Informaticos y Computacion \\ Universitat Politècnica de València \\ Camino de Vera S/N 46022 Valencia (Spain) \\ \{jpalanca, sheras, vbotti, vinglada\}@dsic.upv.es
}

\section{Introduction}

Over the last few years, the emergence of social networks has changed the main activities performed by users on the Internet, going from a mere search and navigation over stored information to a direct interaction with other users. Users have evolved from being consumers of information to real producers (what is known as the transition from Web 1.0 to Web 2.0). Due to the increasing number of heterogeneous users and information that is generated, their unpredictable behavior and the high dynamism of the network structure, users have to cope with a high degree of uncertainty when choosing who to interact to or what information to consume [1]. In order to deal with this uncertainty, users require tools that help them to make decisions regarding their activities within the network. Recommendation systems [2] [3], which are systems that provide effective recommendations about what action users can take or what information they can consume, can be effective tools for performing theses decision-support tasks.

In this paper, we present a persuasive social recommendation system for recipe recommendation in a social network (called receteame.com). The proposed system allows the recommendation of recipes taking into account aspects like persuasion, similarity, friendship, trust, reputation and user food tastes.

\section{Main Purpose: Recommendation in social networks}

Traditional recommender systems base their recommendations on quantitative measures of similarity between the user's preferences and the current items to recommend (i.e. content-based recommenders [4]), between the user's profile and the profile of other users with similar preferences (i.e. collaborative filtering recommenders [5]) and on combinations of both (i.e. hybrid recommenders [6]). However, [7] has stated the inability of current recommender systems to use the large amount of qualitative data available online to empower recommendations. Usually, recommender systems do not provide an explanation about the reasoning process that has been followed to come up with specific recommendations. Recommendations tend to come directly from the recommendation algorithm that runs the website and not from the acquaintances that a user has in his 
social network. However, this does not follow current trends on the Web, where discovering is becoming social and recommendations could be expected to come directly from acquaintances in a decentralised way. Moreover, people trust recommendations more when the engine can provide reasons for them [8]. Thus, what is understood as a good recommendation is changing from the one that minimises some error evaluation to the one that really makes people happier.

In addition, online recommender systems suffer from problems inherent to their use in complex social networks, where the number of users and/or items to recommend can be very high. In the case of collaborative filtering, for instance, the process for comparing two users with the aim of extracting their similarity requires that they have qualified the same objects, which can be unreaslistic in large social networks. Another major weakness of online recommender systems is their trustworthiness. In an open network with a large number of users it is impossible to ensure that all views expressed are true opinions of users and there is no tampering with the resulting recommendations. In order to overcome these problems, it is necessary to embed a social layer in current recommender approaches, taking into account aspects such as the generation of arguments that support recommendations, reputation and trust. Therefore, there are a number of open challenges for the development of a new generation of recommender systems [7], such as exposing underlying assumptions behind recommendations, approaching trust and trustworthiness from the perspective of backing recommendations and providing rationally compelling arguments for recommendations. Our work involves a contribution in these areas, presenting a persuasive social recommendation system for recipe recommendation in a social network.

\section{Demonstration: receteame.com}

receteame. $\mathrm{com}^{1}$ is a website that uses a persuasive social recommendation system to recommend recipes customized for each user. The system retrieves recipes from the Internet, automatically calculates their nutritional information and dietary restrictions and uses this information to make recommendations. The site runs an intelligent algorithm (based on argumentation techniques and social network analysis) to learn the tastes and needs of each user and recommend fully customized recipes. receteame.com is able to learn user preferences from two main sources of information: from the votes that users give to each recipe, and, if the user is registered within Facebook, from the activity of the user and friends, and thus can propose new recipes that the user may like.

Once registered in the system, the user can use the "Recommend me" button for obtaining a recommendation that matches the user's taste and dietary restrictions. This action launches the persuasive social recommendation algorithm that implements the main recommendation functionality of the website. The algorithm receives a recipe recommendation query for a specific user, with a footprint that can include paremeters describing the user profile (preferences

\footnotetext{
${ }^{1}$ http://www.receteame.com; http://buscador.receteame.com
} 
and tastes, dietary restrictions, etc.) and the context of the query (e.g. if the user is looking for a main course, the number of dinner guests, etc.). With this query and the recipe footprint, the algorithm performs two main searches to select a potential set of recipes to recommend to the user. On the one hand, the algorithm follows a content-based recommendation approach to generate a list of recipes that match the recipe footprint. However, note that the accuracy of recommendations generated by this process completely relies on the amount and accuracy of previous votes that the user made to recipes with similar footprints. Therefore, it is highly influenced by the cold start problem (i.e. new users do not have rated recipes) and the drawbacks of applying traditional recommendation approaches on large social networks. To overcome these problems, the algorithm performs an alternative search that follows a social recommendation approach.

On the other hand, the algorithm selects a set of users of the system (the set of friends of the target user and some randomly selected set to avoid the cold start problem when the target user is new on the system and does not have many friends) and spreads the query to obtain recommendations from these users. Each user that has received the query selects a set of recipes that match the original query from his own set of known recipes (those voted by this user). Then, for each user, this part of the algorithm generates an ordered list of recipes to recommend according to three criteria: 1) the preferences of the user that is being asked for recommendations, for instance, taking into account the votes of the user; 2) the preferences of the target user, for instance, taking into account the votes of the target user to a recipe (if any); and 3 ) previous recommendations. This values calculates the confidence on the recommendation of a recipe according to previous recommendations made between the same pair of users.

With the full set of recommended recipes from other users, the algorithm makes an overall ranking of recipes employing three social criteria parameters: 1) the trust on the user who had recommended a recipe from the point of view of the target user and his friends: this parameter is calculated by using a direct trust evaluation between these two users, and, if any, the trust evaluations of the friends of the target user that are also friends of the user that made the recommendation; 2) the reputation of the user who had recommended a recipe: this is a global parameter calculated by computing the average trust regarding all recommendations made by one user in the network; and 3) the strenght of the friendship between the target user and the user that had recommended the recipe: this parameter is calculated by using several predictive friendship variables [9] and depends on the activity of the target user on the social network where the algorithm operates (e.g. Facebook).

The result of this process is a unique and ordered list of recipes to recommed to the target user. Finally, the algorithm mixes the recommendations that has obtained from both searches, assigning weights to ponderate content-based and social recommendations, and selects the best recommnedation to propose. This process also includes an internal agreement procedure based on argumentation techniques, which allows the algorithm to promote those recommendations that come from users that are able to provide better justifications for them [10]. 


\section{Conclusions}

This work presents a persuasive social recommendation system for the recommendation of recipes. The system is embedded in the website receteame.com that is active on the Internet and has more than 2.000 registered users and 30.000 recipe views up to date. We are currently working on making the recommendation process faster and increasingly more accurate and on adding new functionalities to the website. For example, allowing users to add recipes or their own versions of recipes that are already on the web. We will also gradually introduce a more comprehensive dietary restrictions to include those diseases and intolerances that are not yet available. receteame.com may be able to recommend full menus in the future, such as a weekly menu for a family that takes into account the preferences of all members, or a menu for a dinner with friends where one can cook something with confidence that it will like to all guests.

Acknowledgments: This work was partially supported by MINECO/FEDER TIN2012-36586-C03-01 of the Spanish government.

\section{References}

1. van der Aalst, W.M.P., Song, M.: Mining Social Networks: Uncovering Interaction Patterns in Business Processes. In: 2nd Intenational Conference on Business Process Management. Volume 3080 of Lecture Notes in Computer Science., Springer Berlin Heidelberg (June 2004) 244-260

2. Adomavicius, G., Tuzhilin, A.: Toward the Next Generation of Recommender Systems: A Survey of the State-of-the-Art and Possible Extensions. IEEE Transactions on Knowledge and Data Engineering 17(6) (2005) 734-749

3. Zhou, X., Xu, Y., Li, Y., Josang, A., Cox, C.: The state-of-the-art in personalized recommender systems for social networking. Artificial Intelligence Review 37(2) (2012) 119-132

4. Pazzani, M., Billsus, D. In: Content-Based Recommendation Systems. Volume 4321 of Lecture Notes in Computer Science. Springer-Verlag (2007) 325-341

5. Schafer, J., Frankowski, D., Herlocker, J., Sen, S. In: Collaborative Filtering Recommender Systems. Volume 4321 of Lecture Notes in Computer Science. SpringerVerlag (2007) 291-324

6. Burke, R.: Hybrid Recommender Systems: Survey and Experiments. User Modeling and User-Adapted Interaction 12(4) (2002) 331-370

7. Chesñevar, C., Maguitman, A., González, M. In: Empowering Recommendation Technologies Through Argumentation. Springer (2009) 403-422

8. Linden, G., Hong, J., Stonebraker, M., Guzdial:, M.: Recommendation Algorithms, Online Privacy and More. Communications of the ACM 52(5) (2009)

9. Fogués, R., Such, J., Espinosa, A., García-Fornés, A.: A Tool for Retrieving Meaningful Privacy Information from Social Networks. In: Infrastructures and Tools for Multiagent Systems, ITMAS, Editorial Universitat Politècnica de València (2012) $37-50$

10. Heras, S., Navarro, M., Botti, V., Julián, V.: Applying Dialogue Games to Manage Recommendation in Social Networks. In: 6th International Workshop on Argumentation in Multi-Agent Systems, ArgMAS-09. Volume 6057 of LNAI. (2010) $256-272$ 